\title{
The bryophyte flora of nature reserves in central Chile. 1. The moss flora of Los Ruiles Nature Reserve, near Talca
}

\author{
Frank Müller ${ }^{1}$ and Iris Pereira ${ }^{2}$ \\ ${ }^{1}$ Institut für Botanik, Technische Universität Dresden, Mommsenstrasse 13, D-01062 \\ Dresden, Germany; e-mail: fmueller@rcs.urz.tu-dresden.de \\ 2 Instituto de Biología Vegetal y Biotecnología, Universidad de Talca, Casilla 747, Talca, \\ Chile; e-mail: ipereira@utalca.cl
}

\begin{abstract}
The moss flora of the Los Ruiles Nature Reserve, Maule region (VII), central Chile was investigated. Los Ruiles is a small forest reserve dominated by Nothofagus trees and surrounded by plantations of Pinus radiata. The moss species list contains 72 taxa, among which are 36 species newly reported for the Maule region. Several species reach their northernmost known limit in the reserve, including Achrophyllum magellanicum var. magellanicum, Ancistrodes genuflexa, Cryphaea consimilis, Dendrocryphaea lechleri, Lembophyllum orbiculatum, Leptostomum menziesii, Symblepharis krausei, and Zygodon papillatus. To ensure the survival of these rare or local bryophytes, an increase of the proportion of Nothofagus trees in the forests surrounding the reserve is desirable.
\end{abstract}

\section{Introduction}

During the past few years we have investigated the bryophyte flora of several nature reserves in the Maule (VII) region, which is situated in central Chile about $250 \mathrm{~km}$ south of the capital Santiago de Chile. This region is characterized by strong human influence, and large parts of the area, especially in the central Chilean valley, are now under cultivation. Arable land, vineyards, fruit plantations and settlements predominate. In the area of the coast range, the formerly dominant Nothofagus forests have been almost completely cleared and replaced by cultivated land or plantations of Eucalyptus globulus and Pinus radiata. In the Andean range the situation is more favorable, and larger stands of the natural forest vegetation survive, at least in the more remote, deep valleys. However, Nothofagus forests are protected within the region only in few natural reserves, and the number of reserves in the region is low (only seven protected areas). A small area of native Nothofagus forest in the coast range is protected within the Los Ruiles Nature Reserve. During excursions by the authors in March 1999 and March 2000 the moss flora of this nature reserve were studied in detail. 
The moss flora of the Maule region of Chile is still insufficiently known. In the checklist of the mosses of Chile (He 1998) only 65 moss species are listed from this region. In contrast, between 190 and 450 species are known from regions to the south of Maule.

Our investigations had two objectives:

- to increase the knowledge of the bryophyte flora of the up to now poorly investigated Maule region of Chile

- to find out the importance of the few remaining stands of natural forest vegetation for the bryophyte flora of the region and to make proposals for protection measures.

\section{The area of investigation}

The Reserva Nacional Los Ruiles is situated in the Maule (VII) region of Chile, in the coastal range in the province of Cauquenes (Fig. 1), and the following information is taken mostly from the reserve management plan (Cunazza et al. 1995). The reserve was created in 1982, and covers 45.4 ha. It consists of two parts: the Los Ruiles sector, 29 ha in size, situated in the Chanco municipality (72³0’30" W, 3549'30" $\mathrm{S})$, and the El Fin sector, 16.4 ha in size, situated in the Empedrado municipality $\left(72^{\circ} 21^{\prime} 0^{\prime \prime} \mathrm{W}\right.$, $\left.35^{\circ} 37^{\prime} 30^{\prime \prime S}\right)$. The Los Ruiles sector lies in the valley of the Curanilahue River, and the El Fin sector in the valley of the Pino Talca River, two shallow notched valleys in the mountains of the coast range which drain to the Pacific. The Curanilahue River flows throughout the year, but the Pino Talca River is mostly dry during the summer.

Dominant in the reserve are flat to moderately steep slopes with S to SW aspect, which ascend to $810 \mathrm{~m}$ in the Cerro Name.

The soils, derived from the underlying granite and pre-Cambrian metamorphosed sedimentary rocks, are acidic throughout, and can be classified as transitional brown-earth to reddishbrown lateritic soils. They are immature, shallow-layered, well drained and highly vulnerable to erosion.
The macroclimate shows a mediterranean subhumid tendency (Castri \& Hajek 1976). It is characterized by hot dry summers and rainy mild winters. The annual rainfall is about $800 \mathrm{~mm}$. The annual average temperature is $13.8^{\circ} \mathrm{C}$ with maximums of $18.7^{\circ} \mathrm{C}$ in the months of January and February. The coldest months are July and August with average temperatures of $5.9^{\circ} \mathrm{C}$ (Hajek \& Di Castri 1975).

The whole reserve is covered by forest. The dominant forest type is the summer-green Chilean mountain forest of the coast range („Bosque Cudacifolio Maulino“), described as the Nothofagus glauca-Azara petiolaris association. The forest is very rich in woody species, with about 80 shrub and tree species reported. Notable is the occurrence of several plant species at their northernmost known limit, such as Weinmannia trichosperma and Embothrium coccineum. Dominant trees include Nothofagus dombeyi, N. glauca, N. leonii, N. alessandrii, Luma apiculata, Podocarpus saligna, Lithraea caustica, Peumus boldus and Quillaja saponaria. Some vascular plants of the area are on the red list of threatened plants of Chile (Benoit 1989): Nothofagus alessandrii and Pitavia punctata are threatened by extinction, Nothofagus glauca and N. leonii are endangered and Citronella mucronata is potentially endangered because of its rarity. The occurrence of these rare and endangered species was the main reason for the creation of the Los Ruiles Nature Reserve.

\section{Annotated species list}

The species found in the reserve are listed in alphabetical order in Table 1. The nomenclature mainly follows He (1998). For each species the following information is given: distribution in the reserve (occurrence in the Los Ruiles and El Fin sectors), habitat, collection number, distribution in Chile, worldwide range, new record for the Maule region, northernmost known occurrence in Chile. Information on species distribution in Chile is mainly taken from 


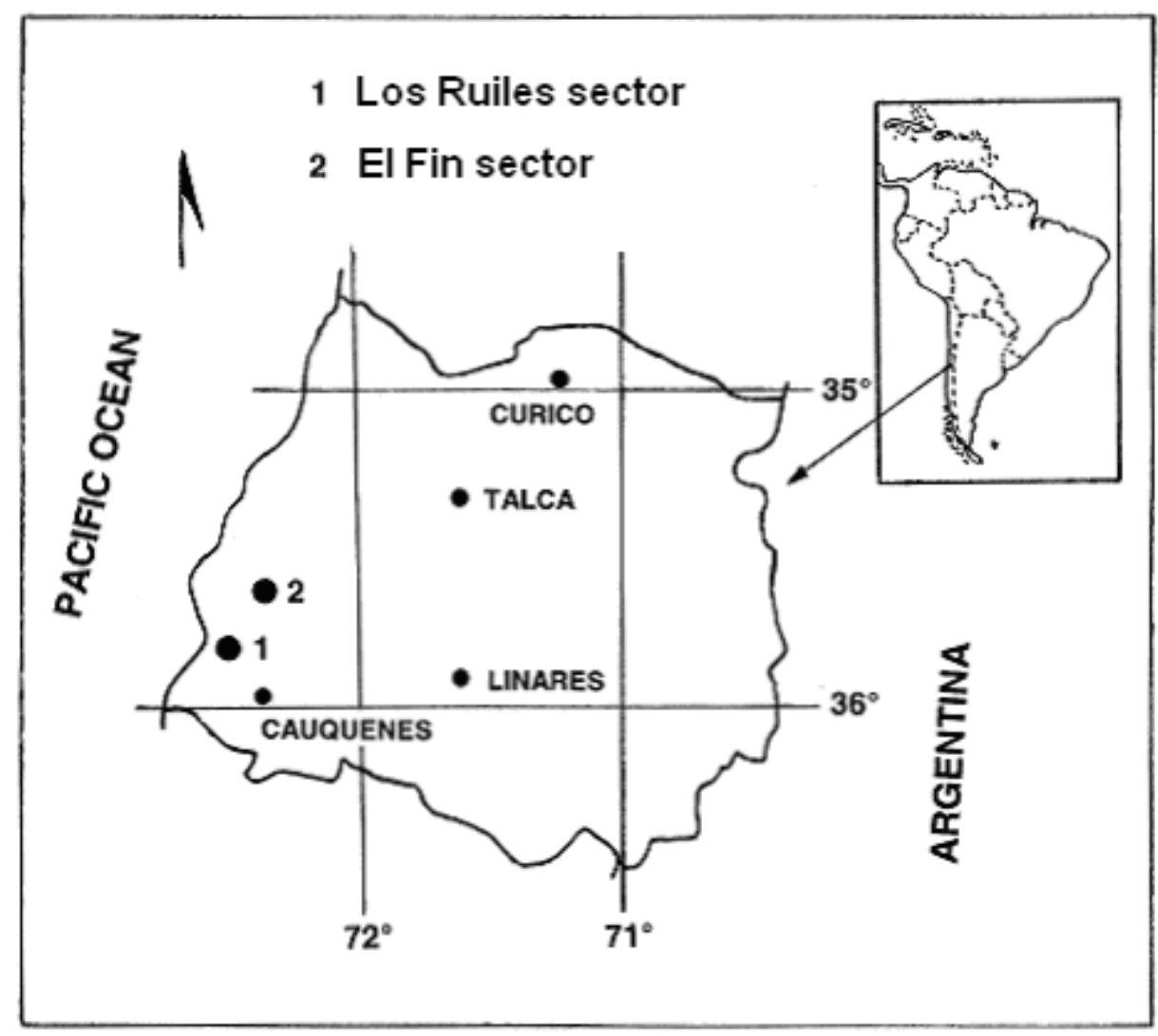

Fig. 1: Geographical position of the studied location

He (1998). (I ... XII = numbers of the administrative regions of Chile, $\mathrm{M}=$ Metropolitan region, JF = Juan Fernandez Is.). The herbarium specimens from our collections are deposited at the herbarium of the Institute of Botany of the University of Dresden (DR).

Taxonomically uncertain and therefore not treated in the list are one sterile sample of Macromitrium and all material of the genera Tortula and Syntrichia.

Besides the mosses, some liverworts were also collected. Among the collections there is material of the following species: Aphanolejeunea asperrima (Steph.) Steph., Blepharolejeunea securifolia (Steph.) R.M.Schust, Cephaloziella subpapillosa Herzog, Cololejeunea minutissima subsp. myriocarpa (Nees \& Mont.) R.M.Schust., Jamesoniella colorata (Lehm.) Schiffn., Lejeunea hahnii Solari, Lepidozia chordulifera Taylor, Lophocolea muricata (Lehm.) Nees, Marchantia berteroana Lehm. \& Lindenb., Noteroclada confluens Taylor ex Hook. \& Wilson, Porella chilensis (Lehm. \& Lindenb.) Trevis., Porella recurva (Taylor) Kühnemann, Radula striata Mitt. ex Steph., Radula tectiloba Steph., Symphyogyna circinata Nees \& Mont. and $S$. rubritincta A.Evans. 


\section{Discussion}

In the reserve, the most important moss habitats are the bark of trees, and rocks and earthy slopes along paths. The forest ground itself is mostly free of mosses because of the dense shade, and mosses are characteristically rather more frequent only on damp forest ground along brooks, where such species as Achrophyllum magellanicum var. magellanicum, Fissidens crispus and Hypnodendron microstictum occur.

The moss diversity on decaying wood was rather low, and all species found on this substrate (e.g., Catagioniopsis berteroana, Hypnum chrysogaster, Juratzkaea seminervis subsp. seminervis, Ptychomitrium fernandesianum, Rigodium toxarium var. toxarium) also occurred in other habitats.

A total of 38 species were found on rocks. Typical mosses on rocks were Amphidium tortuosum, Catagonium nitens, Fissidens oblongifolius, Grimmia trichophylla, Porothamnium panduraefolium, Ptychomitrium fernandesianum, Rigodium toxarium var. toxarium. On walls, Philonotis krausei and the cosmopolitan species Didymodon vinealis and Funaria hygrometrica were found.

A few species occurred on boulders along brooks, submerged or subject to periodic inundation: Dendrocryphaea lechleri, Racomitrium lamprocarpum, Schistidium falcatum, Symplepharis krausei, Vittia pachyloma, Distichophyllum krausei, Achrophyllum magellanicum var. magellanicum, Fissidens rigidulus, and Pohlia wahlenbergii.

The epiphytic vegetation was not very rich in comparison with other areas of the coast range, e.g., the Los Queules nature reserve near Pelluhue. The reason for this is the form of the valleys of the Los Ruiles reserve, which are not narrow and deep, so that the humidity is not constantly high. For this reason, some hygrophilic epiphytes are missing from the reserve, e.g., the broad-leaved Schimperobryum splendidissimum var. splendidissimum which occurs in the adjacent Los Queules reserve on shaded rocks and at the bases of tree trunks. Altogether 31 epiphytic species were found. The most dominant epiphytic mosses of the reserve were: Lembophyllum orbiculatum, Chorisodontium aciphyllum, Glyphothecium gracile, Hypnum chrysogaster, Juratzkaea seminervis subsp. seminervis, Leptodon smithii, Lepyrodon hexastichus, L. parvulus, Neckera chilensis, N. scabridens, Pentastichella pentasticha, Rigodium toxarium var. toxarium and Zygodon intermedius.

Several small brooks, which flow only periodically, drain from the slopes in direction of the main brook. Along these brooks, comparatively damp shady forests have developed, whose epiphytic moss flora is clearly different from the dry slopes outside the valleys. The epiphytic species Dendrocryphaea gorveana and Weymouthia mollis are confined to these areas.

The species diversity of earthy slopes, e.g. alongside paths, is relatively high. The most important species of this substrate type were: Bartramia ambigua, B. ithyphylloides, Breutelia subplicata, Campylopus chilensis, Chrysoblastella chilensis, Ditrichum difficile, Eurhynchiella acanthophylla, Eustichia longirostris, Fissidens oblongifolius, Oligotrichum canaliculatum, Philonotis krausei, Polytrichum juniperinum and Pyrrhobryum mnioides.

The moss flora of the reserve includes both tropical/warm-temperate species which reach their southernmost known distribution limit in the area, as well as Southern Hemisphere/ southern Patagonian species, which reach in the area their northernmost known distribution limit.

The group of tropical / warm-temperate species is represented in the reserve by only a few species, e.g., Fissidens asplenioides, F. crispus and Macrocoma sullivantii. In contrast, the group of Southern Hemisphere/Patagonian elements is represented in the area by many species. Over the half of the mosses of the reserve belong to this distribution type, e.g., Achrophyllum magellanicum var. magellanicum, 
Aongstroemia gayana, Breutelia subplicata, Lembophyllum orbiculatum, Chrysoblastella chilensis, Dendrocryphaea gorveana, D. lechleri, Eurhynchiella acanthophylla, Fissidens oblongifolius, Glyphothecium gracile, Hypnum chrysogaster, Lepyrodon hexastichus, Oligotrichum canaliculatum, Pentastichella pentasticha, Philonotis krausei, Porothamnium panduraefolium,

Ptychomitrium fernandesianum, and Sematophyllum uncinatum. Within this group especially noteworthy are those Patagonian endemics which reach their northernmost limit in the Los Ruiles reserve. On the basis of currently known distribution patterns, this group includes Achrophyllum magellanicum var. magellanicum, Ancistrodes genuflexa, Breutelia subplicata (northernmost record on the Chilean mainland), Lembophyllum orbiculatum, Campylopus chilensis, Catagonium nitens subsp. nitens, Chrysoblastella chilensis, Cryphaea consimilis, Cryphaeophilum molle, Dendrocryphaea gorveana, D. lechleri, Leptostomum menziesii, Lepyrodon patagonicus, Porothamnium panduraefolium, Symblepharis krausei and Zygodon papillatus.

A number of widely distributed species were also found in the reserve, including the cosmopolitan or subcosmopolitan species Ceratodon purpureus, Didymodon vinealis, Funaria hygrometrica, Grimmia trichophylla, Pohlia wahlenbergii, Polytrichum juniperinum, which are also widely distributed in South America.

The Los Ruiles nature reserve is characterized by a species-rich moss flora. Altogether 72 species were found. Particularly important for nature conservation is the occurrence of Patagonian endemics at their northern distribution limit, and these species are especially in need of protection. A red list of the bryophytes of Chile does not yet exist. However, our current knowledge of the Chilean moss flora indicates that some of the species of the reserve are rare and endangered (at least in the Maule region) due to the high influence of man on the landscape. The aim of the management of the reserve should be to cease forestry operations, allowing the proportion of old trees and decaying wood to increase, and maintainting the humid microclimate in valleys along streams. The reserve is very small and therefore highly influenced by marginal disturbances. An enlargement of the reserve and an increase of the proportion of Nothofagus trees in the surrounding forests is urgently needed for the protection of the interesting moss flora of the reserve.

\section{Acknowledgments}

Fieldwork of F. Müller in Chile was supported by a travel fund from the German Academic Exchange Service (DAAD). For the determination or confirmation of specimens we wish to thank K. Yamada (Funaeyama), J.-P. Frahm (Bonn), R. Ochyra (Krakow), R. Pursell (Pennsylvania State University) and A. Touw (Leiden). M.J. Wigginton (Peterborough) kindly checked the manuscript linguistically and made helpful comments.

\section{References}

Benoit, I.L. 1989. Libro Rojo de la flora terrestre de Chile (Primera Parte). - Santiago de Chile. CONAF. 157 pp.

Cunazza, C., Mourgues, V., Villa, A., Yañes, A. \& Torres, J. 1995. Plan de manejo Reserva Nacional Los Ruiles. - Ministerio de Agricultura, Corporacion Nacional Forestal, Region del Maule.

Di Castri, F. \& Hajek, E. 1976. Bioclimatología de Chile. Imprenta Universidad Católica de Chile. Santiago. 107 pp.

Hajek, E. \& Di Castri, F. 1975. Bioclimatographia de Chile. - Santiago, Universidad Catolica de Chile, Dirección de investigación. VRA (Vice-Rectoria Academia), $107 \mathrm{pp}$.

He, S. 1998. A checklist of the mosses of Chile. Journal of the Hattori Botanical Laboratory 85: 103-189. 
Table 1: List of the moss species recorded in the Los Ruiles nature reserve

\begin{tabular}{|c|c|c|c|c|c|c|c|c|}
\hline Species & $\begin{array}{l}\text { Los } \\
\text { Rui } \\
\text {-les }\end{array}$ & $\begin{array}{l}\text { El } \\
\text { Fi } \\
\text { n }\end{array}$ & Habitat & $\begin{array}{l}\text { Collectio } \\
\text { n number }\end{array}$ & $\begin{array}{l}\text { Distributi } \\
\text { on in } \\
\text { Chile }\end{array}$ & Worldwide range & $\begin{array}{l}\text { New } \\
\text { record } \\
\text { s for } \\
\text { the } \\
\text { Maule } \\
\text { region }\end{array}$ & $\begin{array}{l}\text { Northern } \\
\text {-most } \\
\text { known } \\
\text { occurren } \\
\text { ce in } \\
\text { Chile }\end{array}$ \\
\hline $\begin{array}{l}\text { Achrophyllum } \\
\text { magellanicum var. } \\
\text { magellanicum } \\
\text { Besch. }\end{array}$ & $\mathrm{x}$ & $\mathrm{x}$ & $\begin{array}{l}\text { on wet rocks } \\
\text { and ground } \\
\text { near a brook }\end{array}$ & $\begin{array}{l}\text { C803, } \\
\text { C1011 } \\
\text { C1018 } \\
\text { C1062 }\end{array}$ & $\begin{array}{l}\text { VIII, IX, } \\
\text { X, XI, XII }\end{array}$ & $\begin{array}{l}\text { Patagonian } \\
\text { endemic }\end{array}$ & $\mathrm{x}$ & $\mathrm{x}$ \\
\hline $\begin{array}{l}\text { Amphidium } \\
\text { tortuosum } \\
\text { (Hornsch.) H.Rob. }\end{array}$ & $\mathrm{x}$ & $\mathrm{x}$ & $\begin{array}{l}\text { on soil along } \\
\text { a slope, } \\
\text { shaded and } \\
\text { wet rocks }\end{array}$ & $\begin{array}{l}\text { C131, } \\
\text { C1009, } \\
\text { C1016, } \\
\text { C1079 }\end{array}$ & $\begin{array}{l}\text { M, V, VIII, } \\
\text { IX, X, XI, } \\
\text { XII, JF }\end{array}$ & $\begin{array}{l}\text { Pantropical } \\
\text { (Africa, Papua } \\
\text { New Guinea, } \\
\text { Neotropics) to } \\
\text { circum- } \\
\text { subantarctic (S } \\
\text { Africa, Australia, } \\
\text { New Zealand, } \\
\text { southern S } \\
\text { America) }\end{array}$ & $\mathrm{x}$ & \\
\hline $\begin{array}{l}\text { Ancistrodes } \\
\text { genuflexa } \\
\text { (Müll.Hal.) } \\
\text { Crosby }\end{array}$ & & $\mathrm{x}$ & epiphytic & - & IX, X, XI & $\begin{array}{l}\text { Patagonian } \\
\text { endemic }\end{array}$ & $\mathrm{x}$ & $\mathrm{x}$ \\
\hline $\begin{array}{l}\text { Aongstroemia } \\
\text { gayana (Mont.) } \\
\text { Müll.Hal. }\end{array}$ & $\mathrm{x}$ & & $\begin{array}{l}\text { rocks along } \\
\text { a street }\end{array}$ & C180 & $\begin{array}{l}\text { M, V, VII, } \\
\text { VIII, IX, } \\
\text { X, XI }\end{array}$ & $\begin{array}{l}\text { Patagonian } \\
\text { endemic }\end{array}$ & & \\
\hline $\begin{array}{l}\text { Bartramia } \\
\text { ambigua Mont. }\end{array}$ & $\mathrm{x}$ & $\mathrm{x}$ & $\begin{array}{l}\text { earth- } \\
\text { covered } \\
\text { slopes in the } \\
\text { forest, } \\
\text { boulders, } \\
\text { shaded } \\
\text { rocks, forest } \\
\text { floor, wet } \\
\text { rocks near a } \\
\text { brook }\end{array}$ & $\begin{array}{l}\text { C171, } \\
\text { C812, } \\
\text { C818, } \\
\text { C983, } \\
\text { C1020, } \\
\text { C1088 }\end{array}$ & $\begin{array}{l}\text { M, IV, V, } \\
\text { VI, VII, } \\
\text { VIII, IX, } \\
\text { X, XI, XII, } \\
\text { JF }\end{array}$ & S America & & \\
\hline $\begin{array}{l}\text { Bartramia } \\
\text { ithyphylloides } \\
\text { Schimp. ex } \\
\text { Müll.Hal. }\end{array}$ & $\mathrm{x}$ & $\mathrm{x}$ & $\begin{array}{l}\text { slopes along } \\
\text { a path, } \\
\text { shaded } \\
\text { slopes, wet } \\
\text { rocks near a } \\
\text { brook }\end{array}$ & $\begin{array}{l}\text { C777, } \\
\text { C983, } \\
\text { C1053 }\end{array}$ & $\begin{array}{l}\text { M, VIII, } \\
\text { IX, X, XI, } \\
\text { XII, JF }\end{array}$ & $\begin{array}{l}\text { Patagonian } \\
\text { endemic }\end{array}$ & $\mathrm{x}$ & \\
\hline $\begin{array}{l}\text { Breutelia } \\
\text { subplicata } \text { Broth. }\end{array}$ & $\mathrm{x}$ & & $\begin{array}{l}\text { dry rockface } \\
\text { in a valley, } \\
\text { shaded } \\
\text { rocks, } \\
\text { shaded } \\
\text { slopes, } \\
\text { slopes along } \\
\text { a path, rock- } \\
\text { face along a } \\
\text { street }\end{array}$ & $\begin{array}{l}\text { C175, } \\
\text { C778, } \\
\text { C789, } \\
\text { C820, } \\
\text { C1041 }\end{array}$ & $\begin{array}{l}\text { VIII, IX, } \\
\text { X, XI, XII, } \\
\text { JF }\end{array}$ & $\begin{array}{l}\text { Patagonian } \\
\text { endemic }\end{array}$ & $\mathrm{x}$ & $\mathrm{x}$ \\
\hline $\begin{array}{l}\text { Bryum billardieri } \\
\text { Schwägr. }\end{array}$ & $\mathrm{x}$ & & $\begin{array}{l}\text { rock-face } \\
\text { along a } \\
\text { street }\end{array}$ & $\mathrm{C} 126$ & $\begin{array}{l}\text { M, IV, V, } \\
\text { VII, VIII, } \\
\text { IX, X, JF }\end{array}$ & $\begin{array}{l}\text { pantropical to } \\
\text { subtropical, } \\
\text { southwards to New } \\
\text { Zealand, Australia, } \\
\text { southern S }\end{array}$ & & \\
\hline
\end{tabular}




\begin{tabular}{|c|c|c|c|c|c|c|c|c|}
\hline & & & & & & America & & \\
\hline $\begin{array}{l}\text { Campylopus } \\
\text { chilensis De Not. }\end{array}$ & $\mathrm{x}$ & $x$ & $\begin{array}{l}\text { on ground in } \\
\text { the forest, } \\
\text { on dry earth } \\
\text { covered } \\
\text { slopes }\end{array}$ & $\begin{array}{l}\text { C836, } \\
\text { C1030 }\end{array}$ & X, XI, XII & $\begin{array}{l}\text { Patagonian } \\
\text { endemic }\end{array}$ & $x$ & $\mathrm{x}$ \\
\hline $\begin{array}{l}\text { Campylopus } \\
\text { clavatus } \\
\text { (R.Brown) } \\
\text { Hook.f. \& Wilson }\end{array}$ & $x$ & & $\begin{array}{l}\text { rock-face } \\
\text { along a } \\
\text { street }\end{array}$ & $\mathrm{C} 176$ & $\begin{array}{l}\text { V, VII, IX, } \\
\text { X, XI, XII, } \\
\text { JF }\end{array}$ & $\begin{array}{l}\text { Circum- } \\
\text { subantarctic; in S } \\
\text { America at the } \\
\text { Pacific west coast } \\
\text { north to Peru, } \\
\text { Bolivia, Ecuador }\end{array}$ & & \\
\hline $\begin{array}{l}\text { Campylopus } \\
\text { incrassatus } \\
\text { Müll.Hal. }\end{array}$ & $\mathrm{x}$ & & $\begin{array}{l}\text { slopes along } \\
\text { a path }\end{array}$ & C1060 & $\begin{array}{l}\text { M, IV, V, } \\
\text { VI, VII, } \\
\text { VIII, IX, } \\
\text { X, XI }\end{array}$ & $\begin{array}{l}\text { Chile, Peru, Bioko, } \\
\text { Ascension I., } \\
\text { Azores, Australia }\end{array}$ & & \\
\hline $\begin{array}{l}\text { Campylopus } \\
\text { introflexus } \\
\text { (Hedw.) Brid. }\end{array}$ & & $\mathrm{x}$ & $\begin{array}{l}\text { base of a } \\
\text { Nothofagus } \\
\text { glauca trunk }\end{array}$ & C1026 & $\begin{array}{l}\text { M, IV, V, } \\
\text { VII, VIII, } \\
\text { IX, X, XI, } \\
\text { XII, JF }\end{array}$ & $\begin{array}{l}\text { Southern } \\
\text { Hemisphere, } \\
\text { introduced in } \\
\text { Europe }\end{array}$ & & \\
\hline $\begin{array}{l}\text { Catagoniopsis } \\
\text { berteroana } \\
\text { (Mont.) Broth. }\end{array}$ & $\mathrm{x}$ & $\mathrm{x}$ & $\begin{array}{l}\text { epiphytic, } \\
\text { on rocks, } \\
\text { slopes along } \\
\text { a path, } \\
\text { decaying } \\
\text { wood }\end{array}$ & $\begin{array}{l}\text { C990, } \\
\text { C1090 }\end{array}$ & $\begin{array}{l}\text { M, IV, V, } \\
\text { VI, VII, } \\
\text { VIII, IX, } \\
\text { X, JF }\end{array}$ & endemic to Chile & & \\
\hline $\begin{array}{l}\text { Catagonium } \\
\text { nitens subsp. } \\
\text { nitens (Brid.) } \\
\text { Cardot }\end{array}$ & $\mathrm{x}$ & & $\begin{array}{l}\text { rock-face } \\
\text { along a } \\
\text { street, } \\
\text { shaded } \\
\text { rocks, } \\
\text { slopes, base } \\
\text { of a trunk }\end{array}$ & $\begin{array}{l}\text { C169, } \\
\text { C810 }\end{array}$ & $\begin{array}{l}\text { VIII, IX, } \\
\text { X, XII, JF }\end{array}$ & $\begin{array}{l}\text { widespread in the } \\
\text { Southern } \\
\text { Hemisphere (S } \\
\text { America, S and E } \\
\text { Africa, Australia, } \\
\text { Tasmania, New } \\
\text { Zealand, New } \\
\text { Guinea etc.) }\end{array}$ & $\mathrm{x}$ & $\mathrm{x}$ \\
\hline $\begin{array}{l}\text { Ceratodon } \\
\text { purpureus } \\
\text { (Hedw.) Brid. }\end{array}$ & $\mathrm{x}$ & & $\begin{array}{l}\text { rockface } \\
\text { along a } \\
\text { street }\end{array}$ & C179 & $\begin{array}{l}\text { IV, V, VII, } \\
\text { VIII, IX, } \\
\text { X, XI, XII, } \\
\text { JF }\end{array}$ & Cosmopolitan & & \\
\hline $\begin{array}{l}\text { Chileobryon } \\
\text { callicostelloides } \\
\text { (Broth. ex Ther.) } \\
\text { Enroth }\end{array}$ & $\mathrm{x}$ & $\mathrm{x}$ & $\begin{array}{l}\text { on rocks in a } \\
\text { valley }\end{array}$ & $\begin{array}{l}\text { C970, } \\
\text { C1019, } \\
\text { C1025, } \\
\text { C1048 } \\
\end{array}$ & $\begin{array}{l}\text { IV, V, VI, } \\
\text { VII, VIII, } \\
\text { IX, X, XI, } \\
\text { XII, JF }\end{array}$ & endemic to Chile & & \\
\hline $\begin{array}{l}\text { Chorisodontium } \\
\text { aciphyllum } \\
\text { (Hook.f. \& } \\
\text { Wilson) Broth. }\end{array}$ & $\mathrm{x}$ & & $\begin{array}{l}\text { epiphytic, } \\
\text { e.g., on } \\
\text { Nothofagus } \\
\text { obliqua }\end{array}$ & $\begin{array}{l}\text { C1042, } \\
\text { C1070 }\end{array}$ & $\begin{array}{l}\text { VIII, IX, } \\
\text { X, XI, XII, } \\
\text { JF }\end{array}$ & $\begin{array}{l}\text { pan-south- } \\
\text { temperate; widely } \\
\text { distributed in } \\
\text { Patagonia and } \\
\text { known elsewhere } \\
\text { from Amsterdam } \\
\text { Is., Falkland Is., } \\
\text { South Georgia, } \\
\text { Antarctica and } \\
\text { New Zealand }\end{array}$ & $x$ & $\mathrm{x}$ \\
\hline $\begin{array}{l}\text { Chrysoblastella } \\
\text { chilensis (Mont.) } \\
\text { Reim. }\end{array}$ & $\mathrm{x}$ & & $\begin{array}{l}\text { on earth } \\
\text { along } \\
\text { slopes, } \\
\text { rockface } \\
\text { along a } \\
\text { street, }\end{array}$ & $\begin{array}{l}\text { C127, } \\
\text { C776, } \\
\text { C782, } \\
\text { C842 }\end{array}$ & & $\begin{array}{l}\text { Southern } \\
\text { Hemisphere (New } \\
\text { Zealand, Australia, } \\
\text { Marion Island, S } \\
\text { America); in S } \\
\text { America } \\
\end{array}$ & $\mathrm{x}$ & $\mathrm{x}$ \\
\hline
\end{tabular}




\begin{tabular}{|c|c|c|c|c|c|c|c|c|}
\hline & & & $\begin{array}{l}\text { shaded rocks } \\
\text { and slopes }\end{array}$ & & & $\begin{array}{l}\text { northwards to } \\
\text { Colombia }\end{array}$ & & \\
\hline $\begin{array}{l}\text { Cryphaea } \\
\text { consimilis Mont. }\end{array}$ & $\mathrm{x}$ & $x$ & epiphytic & $\begin{array}{l}\text { C982, } \\
\text { C1046 }\end{array}$ & IX, X, XI & $\begin{array}{l}\text { Patagonian } \\
\text { endemic }\end{array}$ & $\mathrm{x}$ & $\mathrm{x}$ \\
\hline $\begin{array}{l}\text { Cryphaeophilum } \\
\text { molle (Dusen) } \\
\text { M.Fleisch. }\end{array}$ & & $x$ & epiphytic & C969 & $\begin{array}{l}\text { VII, IX, X, } \\
\text { XI }\end{array}$ & $\begin{array}{l}\text { Patagonian } \\
\text { endemic }\end{array}$ & & $\mathrm{x}$ \\
\hline $\begin{array}{l}\text { Dendrocryphaea } \\
\text { gorveana (Mont.) } \\
\text { Paris \& Schimp. }\end{array}$ & $\mathrm{x}$ & & $\begin{array}{l}\text { epiphytic } \\
\text { near a brook }\end{array}$ & C843 & $\mathrm{X}, \mathrm{XI}$ & $\begin{array}{l}\text { Patagonian } \\
\text { endemic }\end{array}$ & $\mathrm{x}$ & $\mathrm{x}$ \\
\hline $\begin{array}{l}\text { Dendrocryphaea } \\
\text { lechleri } \\
\text { (Müll.Hal.) Paris } \\
\text { \& Schimp. }\end{array}$ & $\mathrm{x}$ & $\mathrm{x}$ & $\begin{array}{l}\text { wet boulders } \\
\text { in and near a } \\
\text { brook }\end{array}$ & $\begin{array}{l}\text { C838, } \\
\text { C972, } \\
\text { C1087 }\end{array}$ & IX, X & $\begin{array}{l}\text { Patagonian } \\
\text { endemic }\end{array}$ & $\mathrm{x}$ & $\mathrm{x}$ \\
\hline $\begin{array}{l}\text { Dicranoloma } \\
\text { billardieri (Brid.) } \\
\text { Paris }\end{array}$ & & $x$ & epiphytic & C1027 & $\begin{array}{l}\text { V, IX, X, } \\
\text { XI, XII, JF }\end{array}$ & $\begin{array}{l}\text { widespread in } \\
\text { Southern } \\
\text { Hemisphere }\end{array}$ & $x$ & \\
\hline $\begin{array}{l}\text { Didymodon } \\
\text { vinealis (Brid.) } \\
\text { R.H.Zander }\end{array}$ & $\mathrm{x}$ & $\mathrm{x}$ & $\begin{array}{l}\text { shaded, } \\
\text { earth } \\
\text { covered } \\
\text { wall, on } \\
\text { rocks in a } \\
\text { valley }\end{array}$ & $\begin{array}{l}\text { C832, } \\
\text { C1013 }\end{array}$ & $\begin{array}{l}\text { M, IV, V, } \\
\text { VII, VIII, } \\
\text { IX, X, XI }\end{array}$ & Subcosmopolitan & & \\
\hline $\begin{array}{l}\text { Distichophyllum } \\
\text { krausei (Lorentz) } \\
\text { Cardot }\end{array}$ & $\mathrm{x}$ & & $\begin{array}{l}\text { on rocks in a } \\
\text { brook }\end{array}$ & C1038 & $\begin{array}{l}\text { V, VIII, } \\
\text { IX, X, XI, } \\
\text { XII }\end{array}$ & $\begin{array}{l}\text { Patagonian } \\
\text { endemic }\end{array}$ & $\mathrm{x}$ & \\
\hline $\begin{array}{l}\text { Ditrichum difficile } \\
\text { (Duby) M.Fleisch. }\end{array}$ & $\mathrm{x}$ & & $\begin{array}{l}\text { rockface } \\
\text { along a } \\
\text { street, on } \\
\text { earth on } \\
\text { slopes along } \\
\text { a path }\end{array}$ & $\begin{array}{l}\text { C173, } \\
\text { C845 }\end{array}$ & $\begin{array}{l}\text { VII, VIII, } \\
\text { IX, X, XI, } \\
\text { XII, JF }\end{array}$ & \begin{tabular}{|l} 
Southern \\
Hemisphere (S \\
Africa, SE Asia, \\
Micronesia, \\
Australia, New \\
Zealand, S \\
America)
\end{tabular} & & \\
\hline $\begin{array}{l}\text { Eurhynchiella } \\
\text { acanthophylla } \\
\text { (Mont.) } \\
\text { M.Fleisch. }\end{array}$ & $\mathrm{x}$ & $x$ & $\begin{array}{l}\text { on earth } \\
\text { along } \\
\text { slopes, on } \\
\text { boulders, } \\
\text { epiphytic, } \\
\text { forest floor }\end{array}$ & $\begin{array}{l}\text { C833, } \\
\text { C846, } \\
\text { C1065 }\end{array}$ & $\begin{array}{l}\text { M, IV, V, } \\
\text { VI, VII, } \\
\text { VIII, IX, } \\
\text { X, XI }\end{array}$ & $\begin{array}{l}\text { Patagonian } \\
\text { endemic }\end{array}$ & & \\
\hline $\begin{array}{l}\text { Eustichia } \\
\text { longirostris } \\
\text { (Brid.) Brid. }\end{array}$ & $\mathrm{x}$ & & $\begin{array}{l}\text { slopes along } \\
\text { a path, forest } \\
\text { ground }\end{array}$ & $\begin{array}{l}\text { C1069, } \\
\text { C1086 }\end{array}$ & $\begin{array}{l}\text { M, V, VII, } \\
\text { VIII, IX, } \\
\text { X, XI, XII, } \\
\text { JF }\end{array}$ & $\begin{array}{l}\text { Central and S } \\
\text { America, } \\
\text { Subantarctic } \\
\text { Islands, S Africa, } \\
\text { western Indian } \\
\text { Ocean } \\
\end{array}$ & & \\
\hline $\begin{array}{l}\text { Fissidens } \\
\text { asplenioides } \\
\text { Hedw. }\end{array}$ & $\mathrm{x}$ & & $\begin{array}{l}\text { on rocks } \\
\text { near a } \\
\text { brook, } \\
\text { shaded } \\
\text { slopes } \\
\end{array}$ & C1069 & $\begin{array}{l}\text { V, VII, } \\
\text { VIII, IX, } \\
\text { X, XI, XII, } \\
\text { JF }\end{array}$ & $\begin{array}{l}\text { pan(sub)tropical- } \\
\text { temperate }\end{array}$ & & \\
\hline $\begin{array}{l}\text { Fissidens crispus } \\
\text { Mont. }\end{array}$ & $\mathrm{x}$ & $\mathrm{x}$ & $\begin{array}{l}\text { on earth near } \\
\text { a periodic } \\
\text { brook, } \\
\text { earthy } \\
\text { slopes along } \\
\text { a path }\end{array}$ & $\begin{array}{l}\text { C1032, } \\
\text { C1054 }\end{array}$ & $\begin{array}{l}\text { M, V, VI, } \\
\text { VII, VIII, } \\
\text { IX, X, JF }\end{array}$ & $\begin{array}{l}\text { SW U.S.A., } \\
\text { Mexico, Central } \\
\text { and S America, } \\
\text { West Indies }\end{array}$ & & \\
\hline
\end{tabular}




\begin{tabular}{|c|c|c|c|c|c|c|c|c|}
\hline $\begin{array}{l}\text { Fissidens } \\
\text { oblongifolius } \\
\text { Hook.f. \& Wilson }\end{array}$ & $\mathrm{x}$ & & $\begin{array}{l}\text { on rocks } \\
\text { near a } \\
\text { brook, on } \\
\text { earthy } \\
\text { slopes, rock- } \\
\text { face, shaded } \\
\text { slopes, } \\
\text { shaded } \\
\text { boulders, on } \\
\text { rocks in a } \\
\text { valley } \\
\end{array}$ & $\begin{array}{l}\text { C167, } \\
\text { C799, } \\
\text { C } 840, \\
\text { C1044, } \\
\text { C1047, } \\
\text { C1075 }\end{array}$ & $\begin{array}{l}\text { VII, VIII, } \\
\text { IX, X }\end{array}$ & \begin{tabular}{|l|} 
Southern \\
Hemisphere (New \\
Zealand, Australia, \\
Tasmania, \\
Patagonia)
\end{tabular} & & $\mathrm{x}$ \\
\hline $\begin{array}{l}\text { Fissidens } \\
\text { rigidulus Hook.f. } \\
\text { \& Wilson }\end{array}$ & & $\mathrm{x}$ & $\begin{array}{l}\text { on wet rocks } \\
\text { near a brook }\end{array}$ & C1033 & $\begin{array}{l}\text { M, V, VI, } \\
\text { VII, VIII, } \\
\text { IX, X, XI, } \\
\text { XII, JF }\end{array}$ & $\begin{array}{l}\text { Mexico, Central } \\
\text { and S America, } \\
\text { Malesia, Australia, } \\
\text { New Zealand, SW } \\
\text { Pacific, } \\
\text { Subantarctic } \\
\text { Islands }\end{array}$ & & \\
\hline $\begin{array}{l}\text { Funaria } \\
\text { hygrometrica var. } \\
\text { hygrometrica } \\
\text { Hedw. }\end{array}$ & $\mathrm{x}$ & & $\begin{array}{l}\text { on a wall } \\
\text { near a brook }\end{array}$ & $\mathrm{C} 825$ & $\begin{array}{l}\text { M, IV, V, } \\
\text { VI, VII, } \\
\text { VIII, IX, } \\
\text { X, XI, XII, } \\
\text { JF }\end{array}$ & Cosmopolitan & & \\
\hline $\begin{array}{l}\text { Glyphothecium } \\
\text { gracile (Hampe) } \\
\text { Broth. }\end{array}$ & $\mathrm{x}$ & $\mathrm{x}$ & epiphytic & $\begin{array}{l}\text { C769, } \\
\text { C1008 }\end{array}$ & $\begin{array}{l}\text { V, VI, VII, } \\
\text { VIII, IX, } \\
\text { X, XI, XII }\end{array}$ & $\begin{array}{l}\text { Patagonian } \\
\text { endemic }\end{array}$ & & \\
\hline $\begin{array}{l}\text { Grimmia } \\
\text { trichophylla Grev. }\end{array}$ & $\mathrm{x}$ & $x$ & $\begin{array}{l}\text { on boulders, } \\
\text { dry } \\
\text { rockface, } \\
\text { rocks, } \\
\text { shaded rocks }\end{array}$ & $\begin{array}{l}\text { C128, } \\
\text { C791, } \\
\text { C795, } \\
\text { C1073 }\end{array}$ & $\begin{array}{l}\text { M, IV, V, } \\
\text { VI, VII, } \\
\text { VIII, IX, } \\
\text { X, XI }\end{array}$ & subcosmopolitan & & \\
\hline $\begin{array}{l}\text { Hypnodendron } \\
\text { microstictum Mitt. }\end{array}$ & $\mathrm{x}$ & $\mathrm{x}$ & $\begin{array}{l}\text { on wet rocks } \\
\text { and forest } \\
\text { ground near } \\
\text { a brook }\end{array}$ & C966 & $\begin{array}{l}\text { M, VI, IX, } \\
\text { X, XI, JF }\end{array}$ & $\begin{array}{l}\text { Patagonian } \\
\text { endemic }\end{array}$ & $\mathrm{x}$ & \\
\hline $\begin{array}{l}\text { Hypnum } \\
\text { chrysogaster } \\
\text { Müll.Hal. }\end{array}$ & $\mathrm{x}$ & $\mathrm{x}$ & $\begin{array}{l}\text { epiphytic on } \\
\text { Nothofagus } \\
\text { alessandrii, } \\
N \text {. glauca, } \\
\text { N. obliqua, } \\
\text { on decaying } \\
\text { wood } \\
\end{array}$ & $\begin{array}{l}\text { C807, } \\
\text { C826, } \\
\text { C1010, } \\
\text { C1059 }\end{array}$ & $\mathrm{X}, \mathrm{XI}, \mathrm{JF}$ & $\begin{array}{l}\text { Southern } \\
\text { Hemisphere } \\
\text { (Patagonia, New } \\
\text { Zealand, Australia, } \\
\text { Tasmania) }\end{array}$ & $\mathrm{x}$ & $\mathrm{x}$ \\
\hline $\begin{array}{l}\text { Juratzkaea } \\
\text { seminervis subsp. } \\
\text { seminervis (Kunze } \\
\text { ex Schwägr.) } \\
\text { Lorentz }\end{array}$ & $\mathrm{x}$ & $x$ & $\begin{array}{l}\text { epiphytic, } \\
\text { e.g., on } \\
\text { Nothofagus, } \\
\text { on decaying } \\
\text { wood }\end{array}$ & $\begin{array}{l}\text { C985, } \\
\text { C987, } \\
\text { C997, } \\
\text { C998, } \\
\text { C1004, } \\
\text { C1037 }\end{array}$ & $\begin{array}{l}\text { M, II, IV, } \\
\text { V, VI, VII, } \\
\text { VIII, IX, X }\end{array}$ & endemic to Chile & & \\
\hline $\begin{array}{l}\text { Lembophyllum } \\
\text { orbiculatum } \\
\text { (Thér.) Tangney }\end{array}$ & $\mathrm{x}$ & $\mathrm{x}$ & $\begin{array}{l}\text { epiphytic, } \\
\text { e.g., on } \\
\text { Nothofagus } \\
\text { dombeyi and } \\
\text { on the base } \\
\text { of a trunk }\end{array}$ & C834 & $\begin{array}{l}\text { VIII, IX, } \\
\text { X, XII }\end{array}$ & $\begin{array}{l}\text { Patagonian } \\
\text { endemic }\end{array}$ & $\mathrm{x}$ & $\mathrm{x}$ \\
\hline $\begin{array}{l}\text { Leptodon smithii } \\
\text { (Hedw.) F.Weber } \\
\text { \& D.Mohr }\end{array}$ & $\mathrm{x}$ & $x$ & epiphytic & C829 & $\begin{array}{l}\text { M, V, VII, } \\
\text { VIII, IX, } \\
\text { X, XI, JF }\end{array}$ & $\begin{array}{l}\text { Bipolar (N } \\
\text { America, } \\
\text { Holarctis, } \\
\text { Australia, New }\end{array}$ & & \\
\hline
\end{tabular}

TROPICAL BRYOLOGY 27 (2006) 


\begin{tabular}{|c|c|c|c|c|c|c|c|c|}
\hline & & & & & & $\begin{array}{l}\text { Zealand, S Africa, } \\
\text { S America) }\end{array}$ & & \\
\hline $\begin{array}{l}\text { Leptostomum } \\
\text { menziesii (Hook.) } \\
\text { R.Brown }\end{array}$ & $x$ & & epiphytic & - & $\begin{array}{l}\text { VIII, IX, } \\
\text { X, XII, JF }\end{array}$ & $\begin{array}{l}\text { Patagonian } \\
\text { endemic }\end{array}$ & $\mathrm{x}$ & $\mathrm{x}$ \\
\hline $\begin{array}{l}\text { Lepyrodon } \\
\text { hexastichus } \\
\text { (Mont.) Wijk \& } \\
\text { Margad. }\end{array}$ & $x$ & & epiphytic & $\begin{array}{l}\text { C772, } \\
\text { C797 }\end{array}$ & $\begin{array}{l}\text { VI, VII, } \\
\text { VIII, IX, } \\
\text { X, XI, XII, } \\
\text { JF }\end{array}$ & $\begin{array}{l}\text { Patagonian } \\
\text { endemic }\end{array}$ & & \\
\hline $\begin{array}{l}\text { Lepyrodon } \\
\text { lagurus (Hook..f.) } \\
\text { Mitt. }\end{array}$ & $x$ & & epiphytic & C1077 & $\begin{array}{l}\text { VII, VIII, } \\
\text { IX, X, XI, } \\
\text { XII }\end{array}$ & $\begin{array}{l}\text { Patagonia, South } \\
\text { Georgia }\end{array}$ & & \\
\hline $\begin{array}{l}\text { Lepyrodon } \\
\text { parvulus Mitt. }\end{array}$ & $\mathrm{x}$ & $\mathrm{x}$ & epiphytic & $\begin{array}{l}\text { C971, } \\
\text { C973, } \\
\text { C1077, } \\
\text { C1082, }\end{array}$ & $\begin{array}{l}\text { IV, V, } \\
\text { VIII, IX, } \\
\text { X, JF }\end{array}$ & $\begin{array}{l}\text { Patagonian } \\
\text { endemic }\end{array}$ & $\mathrm{x}$ & \\
\hline $\begin{array}{l}\text { Lepyrodon } \\
\text { patagonicus } \\
\text { (Cardot \& Broth.) } \\
\text { B.H.Allen }\end{array}$ & & $\mathrm{x}$ & $\begin{array}{l}\text { epiphytic on } \\
\text { the base of } \\
\text { trunks }\end{array}$ & C965 & $\begin{array}{l}\text { V, VIII, } \\
\text { IX, X, XI, } \\
\text { XII, JF }\end{array}$ & $\begin{array}{l}\text { Patagonian } \\
\text { endemic }\end{array}$ & $\mathrm{x}$ & \\
\hline $\begin{array}{l}\text { Macrocoma } \\
\text { sullivantii } \\
\text { (Müll.Hal.) Grout }\end{array}$ & $x$ & $\mathrm{x}$ & epiphytic & C1056 & $\begin{array}{l}\text { V, VIII, } \\
\text { IX, X, XII, } \\
\text { JF }\end{array}$ & $\begin{array}{l}\text { SE U.S.A. to } \\
\text { southern S } \\
\text { America, Indian } \\
\text { Subcontinent, } \\
\text { China, E Asia, } \\
\text { North-Central } \\
\text { Pacific }\end{array}$ & $\mathrm{x}$ & \\
\hline $\begin{array}{l}\text { Neckera chilensis } \\
\text { Schimp. }\end{array}$ & $x$ & $\mathrm{x}$ & epiphytic & $\mathrm{C} 800$ & $\begin{array}{l}\text { M, IV, V, } \\
\text { VIII, IX, } \\
\text { X, XI, XII }\end{array}$ & $\begin{array}{l}\text { Central and } \mathrm{S} \\
\text { America }\end{array}$ & $\mathrm{x}$ & \\
\hline $\begin{array}{l}\text { Neckera } \\
\text { scabridens } \\
\text { Müll.Hal. }\end{array}$ & $x$ & $\mathrm{x}$ & epiphytic & C830 & $\begin{array}{l}\text { M, IV, V, } \\
\text { VIII, IX, } \\
\text { X, XI }\end{array}$ & $\begin{array}{l}\text { Central and } \mathrm{S} \\
\text { America }\end{array}$ & $\mathrm{x}$ & \\
\hline $\begin{array}{l}\text { Oligotrichum } \\
\text { canaliculatum } \\
\text { (Hook.f. \& } \\
\text { Arnott) Mitt. }\end{array}$ & $\mathrm{x}$ & & $\begin{array}{l}\text { on earth on } \\
\text { slopes along } \\
\text { a path, rock- } \\
\text { face along a } \\
\text { street }\end{array}$ & $\begin{array}{l}\text { C170, } \\
\text { C845 }\end{array}$ & $\begin{array}{l}\text { M, V, VII, } \\
\text { VIII, IX, } \\
\text { X, XI, JF }\end{array}$ & $\begin{array}{l}\text { Patagonian } \\
\text { endemic }\end{array}$ & & \\
\hline $\begin{array}{l}\text { Orthotheciella } \\
\text { varia (Hedw.) } \\
\text { Ochyra }\end{array}$ & & $\mathrm{x}$ & $\begin{array}{l}\text { on wet rocks } \\
\text { near a brook }\end{array}$ & C1006 & $\begin{array}{l}\text { M, V, VIII, } \\
\mathrm{X}\end{array}$ & Bipolar & $\mathrm{x}$ & \\
\hline $\begin{array}{l}\text { Orthotrichum } \\
\text { bicolor Thér. }\end{array}$ & $\mathrm{x}$ & & epiphytic & C1074 & $\begin{array}{l}\text { V, VII, } \\
\text { VIII, IX, } \\
\text { X, XII }\end{array}$ & $\begin{array}{l}\text { Patagonian } \\
\text { endemic }\end{array}$ & & \\
\hline $\begin{array}{l}\text { Pentastichella } \\
\text { pentasticha } \\
\text { (Mont.) Müll.Hal. }\end{array}$ & $\mathrm{x}$ & $\mathrm{x}$ & $\begin{array}{l}\text { epiphytic, } \\
\text { e.g., on } \\
\text { Nothofagus } \\
\text { dombeyi }\end{array}$ & C828 & $\begin{array}{l}\text { V, VII, } \\
\text { VIII, IX, } \\
\text { X, XI, XII, } \\
\text { JF }\end{array}$ & $\begin{array}{l}\text { southern S } \\
\text { America, Peru }\end{array}$ & & \\
\hline $\begin{array}{l}\text { Philonotis krausei } \\
\text { (Müll.Hal.) Broth. }\end{array}$ & $\mathrm{x}$ & & $\begin{array}{l}\text { on slopes } \\
\text { along a path, } \\
\text { shaded } \\
\text { slopes, } \\
\text { shaded earth } \\
\text { covered wall }\end{array}$ & $\begin{array}{l}\text { C827, } \\
\text { C844, } \\
\text { C1051 }\end{array}$ & $\begin{array}{l}\text { M, V, VI, } \\
\text { VII, VIII, } \\
\text { IX, X, XI, } \\
\text { JF }\end{array}$ & $\begin{array}{l}\text { Patagonian } \\
\text { endemic }\end{array}$ & & \\
\hline $\begin{array}{l}\text { Pohlia } \\
\text { wahlenbergii } \\
\text { (F.Weber \& } \\
\text { D.Mohr) }\end{array}$ & & $\mathrm{x}$ & $\begin{array}{l}\text { on rocks in a } \\
\text { brook }\end{array}$ & C1021 & $\begin{array}{l}\text { M, V, VI, } \\
\text { VII, VIII, } \\
\text { IX, X, XI, } \\
\text { XII }\end{array}$ & subcosmopolitan & & \\
\hline
\end{tabular}




\begin{tabular}{|c|c|c|c|c|c|c|c|c|}
\hline A.L.Andrews & & & & & & & & \\
\hline $\begin{array}{l}\text { Polytrichum } \\
\text { juniperinum } \\
\text { Hedw. }\end{array}$ & $x$ & & $\begin{array}{l}\text { rock-face } \\
\text { along a } \\
\text { street }\end{array}$ & C177 & $\begin{array}{l}\text { M, V, VI, } \\
\text { VII, VIII, } \\
\text { IX, X, XI, } \\
\text { XII }\end{array}$ & subcosmopolitan & & \\
\hline $\begin{array}{l}\text { Porothamnium } \\
\text { panduraefolium } \\
\text { (Müll.Hal.) } \\
\text { M.Fleisch. }\end{array}$ & $x$ & $\mathrm{x}$ & $\begin{array}{l}\text { on shaded } \\
\text { rocks near } \\
\text { brooks }\end{array}$ & $\begin{array}{l}\text { C779, } \\
\text { C783 }\end{array}$ & $\begin{array}{l}\text { VIII, IX, } \\
\text { X, XI, XII }\end{array}$ & $\begin{array}{l}\text { Patagonian } \\
\text { endemic }\end{array}$ & $\mathrm{x}$ & $\mathrm{x}$ \\
\hline $\begin{array}{l}\text { Ptychomitrium } \\
\text { fernandesianum } \\
\text { (Mitt.) A.Jaeger }\end{array}$ & $\mathrm{x}$ & $\mathrm{x}$ & $\begin{array}{l}\text { rock-face } \\
\text { along a } \\
\text { street, on } \\
\text { boulders, on } \\
\text { decaying } \\
\text { wood }\end{array}$ & $\begin{array}{l}\text { C129, } \\
\text { C821, } \\
\text { C989 }\end{array}$ & $\mathrm{X}, \mathrm{JF}$ & $\begin{array}{l}\text { Patagonian } \\
\text { endemic }\end{array}$ & $\mathrm{x}$ & $\mathrm{x}$ \\
\hline $\begin{array}{l}\text { Pyrrhobryum } \\
\text { mnioides } \\
\text { (Hook.f.) Manuel }\end{array}$ & $\mathrm{x}$ & & $\begin{array}{l}\text { slopes along } \\
\text { a path, forest } \\
\text { ground }\end{array}$ & C774 & $\begin{array}{l}\text { M, V, VII, } \\
\text { VIII, IX, } \\
\text { X, XI, XII, } \\
\text { JF }\end{array}$ & \begin{tabular}{|l|} 
Central and S \\
America, \\
Australia, \\
Tasmania, New \\
Zealand \\
\end{tabular} & & \\
\hline $\begin{array}{l}\text { Racomitrium } \\
\text { lamprocarpum } \\
\text { (Müll.Hal.) } \\
\text { A.Jaeger }\end{array}$ & $\mathrm{x}$ & & $\begin{array}{l}\text { on boulders } \\
\text { in and near a } \\
\text { brook }\end{array}$ & $\begin{array}{l}\text { C801, } \\
\text { C839 }\end{array}$ & \begin{tabular}{|l|} 
M, VIII, \\
IX, X, XI, \\
XII, JF
\end{tabular} & $\begin{array}{l}\text { southern S } \\
\text { America }\end{array}$ & $\mathrm{x}$ & \\
\hline $\begin{array}{l}\text { Rhaphidorrhynchi } \\
\text { um callidum } \\
\text { (Mont.) Broth. }\end{array}$ & $\mathrm{x}$ & $\mathrm{x}$ & $\begin{array}{l}\text { epiphytic in } \\
\text { a shaded and } \\
\text { humid forest } \\
\text { near a } \\
\text { brook, on } \\
\text { boulders, } \\
\text { epiphytic on } \\
\text { Nothofagus } \\
\text { glauca }\end{array}$ & $\begin{array}{l}\text { C824, } \\
\text { C1057 }\end{array}$ & $\begin{array}{l}\text { IV, V, VI, } \\
\text { VII, VIII, } \\
\text { IX, X, XI, } \\
\text { XII }\end{array}$ & endemic to Chile & & \\
\hline $\begin{array}{l}\text { Rigodium } \\
\text { toxarium var. } \\
\text { toxarium } \\
\text { (Schwägr.) } \\
\text { A.Jaeger }\end{array}$ & $\mathrm{x}$ & $\mathrm{x}$ & $\begin{array}{l}\text { on decaying } \\
\text { wood, rocks } \\
\text { near a } \\
\text { brook, } \\
\text { epiphytic, } \\
\text { boulders, } \\
\text { boulders } \\
\text { near a brook }\end{array}$ & $\begin{array}{l}\text { C780, } \\
\text { C785, } \\
\text { C804, } \\
\text { C808, } \\
\text { C813, } \\
\text { C967, } \\
\text { C1031, } \\
\text { C1063, }\end{array}$ & $\begin{array}{l}\text { IV, V, VI, } \\
\text { VIII, IX, } \\
\text { X, XI, JF }\end{array}$ & $\begin{array}{l}\text { Central and S } \\
\text { America (from } \\
\text { Mexico to } \\
\text { Patagonia), E } \\
\text { Africa, East } \\
\text { African islands }\end{array}$ & $\mathrm{x}$ & \\
\hline $\begin{array}{l}\text { Schistidium } \\
\text { falcatum (Hook.f. } \\
\text { \& Wilson) Bremer }\end{array}$ & $\mathrm{x}$ & & $\begin{array}{l}\text { boulders } \\
\text { near a brook }\end{array}$ & C816 & X, XI, XII & $\begin{array}{l}\text { amphiatlantic } \\
\text { subantarctic } \\
\text { (Subantarctic } \\
\text { Islands, } \\
\text { Antarctica, Tierra } \\
\text { del Fuego, streets } \\
\text { of Magellan) }\end{array}$ & $\mathrm{x}$ & $\mathrm{x}$ \\
\hline $\begin{array}{l}\text { Sematophyllum cf. } \\
\text { uncinatum } \\
\text { I.G.Stone \& } \\
\text { G.A.M.Scott }\end{array}$ & $\mathrm{x}$ & & $\begin{array}{l}\text { on boulders } \\
\text { near a } \\
\text { brook, on } \\
\text { slopes }\end{array}$ & $\begin{array}{l}\text { C773, } \\
\text { C1068 }\end{array}$ & VI & $\begin{array}{l}\text { New Zealand, } \\
\text { Australia, } \\
\text { Patagonia }\end{array}$ & $\mathrm{x}$ & \\
\hline $\begin{array}{l}\text { Symblepharis } \\
\text { krausei (Lorentz) } \\
\text { Ochyra \& Matteri }\end{array}$ & $\mathrm{x}$ & & $\begin{array}{l}\text { on boulders } \\
\text { in a brook }\end{array}$ & C817 & $\begin{array}{l}\text { X, XI, XII, } \\
\text { JF }\end{array}$ & $\begin{array}{l}\text { Patagonian } \\
\text { endemic }\end{array}$ & $\mathrm{x}$ & $\mathrm{x}$ \\
\hline
\end{tabular}




\begin{tabular}{|c|c|c|c|c|c|c|c|c|}
\hline $\begin{array}{l}\text { Thuidiopsis } \\
\text { sparsa (Hook.f. \& } \\
\text { Wilson) Broth. }\end{array}$ & $\mathrm{x}$ & & on boulders & C793 & $\begin{array}{l}\text { VIII, IX, } \\
\text { X, XI, JF }\end{array}$ & $\begin{array}{l}\text { Malesia, New } \\
\text { Caledonia, } \\
\text { Oceania, } \\
\text { Autralasia, S } \\
\text { America, Comores, } \\
\text { Tristan da Cunha, } \\
\text { Madeira }\end{array}$ & $\mathrm{x}$ & $\mathrm{x}$ \\
\hline $\begin{array}{l}\text { Triquetrella } \\
\text { patagonica } \\
\text { Müll.Hal. }\end{array}$ & & $\mathrm{x}$ & $\begin{array}{l}\text { sun exposed, } \\
\text { earth } \\
\text { covered } \\
\text { rocks in a } \\
\text { clearing of } \\
\text { the forest }\end{array}$ & C1029 & V, VIII, IX & endemic to Chile & $\mathrm{x}$ & \\
\hline $\begin{array}{l}\text { Vittia pachyloma } \\
\text { (Mont.) Ochyra }\end{array}$ & & $\mathrm{x}$ & $\begin{array}{l}\text { wet rocks in } \\
\text { a brook }\end{array}$ & - & $\begin{array}{l}\text { M, V, VI, } \\
\text { VII, VIII, } \\
\text { IX, X, XI, } \\
\text { XII, JF }\end{array}$ & $\begin{array}{l}\text { S America } \\
\text { (Colombia to } \\
\text { Tierra del Fuego), } \\
\text { S Africa }\end{array}$ & & \\
\hline $\begin{array}{l}\text { Weissia } \text { cf. } \\
\text { kunzeana } \\
\text { Müll.Hal. }\end{array}$ & $\mathrm{x}$ & & $\begin{array}{l}\text { slopes along } \\
\text { a path in the } \\
\text { forest }\end{array}$ & $\mathrm{C} 1050$ & VI, X, JF & endemic to Chile & $\mathrm{x}$ & \\
\hline $\begin{array}{l}\text { Weymouthia } \\
\text { mollis (Hedw.) } \\
\text { Broth. }\end{array}$ & $\mathrm{x}$ & $\mathrm{x}$ & $\begin{array}{l}\text { epiphytic in } \\
\text { a humid } \\
\text { forest, } \\
\text { pendulous }\end{array}$ & $\begin{array}{l}\text { C986, } \\
\text { C992, } \\
\text { C1052 }\end{array}$ & $\begin{array}{l}\text { IV, IX, X, } \\
\text { XI, XII, JF }\end{array}$ & $\begin{array}{l}\text { Patagonia, } \\
\text { Australia, } \\
\text { Tasmania, New } \\
\text { Zealand }\end{array}$ & $\mathrm{x}$ & \\
\hline $\begin{array}{l}\text { Zygodon inermis } \\
\text { Malta }\end{array}$ & & $\mathrm{x}$ & epiphytic & C991 & VII, X, XI & $\begin{array}{l}\text { Patagonian } \\
\text { endemic }\end{array}$ & & \\
\hline $\begin{array}{l}\text { Zygodon } \\
\text { intermedius Bruch } \\
\text { \& Schimp. }\end{array}$ & $\mathrm{x}$ & $\mathrm{x}$ & $\begin{array}{l}\text { epiphytic on } \\
\text { Nothofagus } \\
\text { dombeyi and } \\
\text { other trees }\end{array}$ & $\begin{array}{l}\text { C794, } \\
\text { C798, } \\
\text { C1001, } \\
\text { C1035, } \\
\text { C1089, }\end{array}$ & $\begin{array}{l}\text { VII, X, XI, } \\
\text { XII, JF }\end{array}$ & $\begin{array}{l}\text { pantropical- } \\
\text { Southern } \\
\text { Hemisphere }\end{array}$ & & \\
\hline $\begin{array}{l}\text { Zygodon } \\
\text { papillatus Mont. }\end{array}$ & $\mathrm{x}$ & & epiphytic & C1089 & $\begin{array}{l}\text { VIII, IX, } \\
\text { X, XI, XII } \\
\end{array}$ & $\begin{array}{l}\text { Patagonian } \\
\text { endemic }\end{array}$ & $\mathrm{x}$ & $\mathrm{x}$ \\
\hline
\end{tabular}

